\title{
Epidemiology of Musculoskeletal Tuberculosis in an Area with High Disease Prevalence
}

\author{
Michael F. G. Held ${ }^{1}$, Sven Hoppe ${ }^{1}$, Maritz Laubscher ${ }^{1}$, Stewart Mears ${ }^{1}$, \\ Stewart Dix-Peek ${ }^{1}$, Heather J. Zar ${ }^{2}$, Robert N. Dunn ${ }^{1}$ \\ ${ }^{1}$ Orthopaedic Research Unit, Department of Orthopaedic Surgery, Groote Schuur Hospital, University of Cape Town, Cape Town, South Africa \\ ${ }^{2}$ Department of Pediatrics and Child Health, Red Cross War Memorial Children's Hospital, University of Cape Town and \\ MRC Unit on Child \& Adolescent Health, University of Cape Town, Cape Town, South Africa
}

Study Design: Retrospective observational study.

Purpose: The aim of this study was to assess the distribution of age and site of infection in patients with musculoskeletal tuberculosis (TB) and determine the number of TB/human immunodeficiency virus (HIV) coinfections as well as the incidence of multidrugresistant (MDR) TB.

Overview of Literature: Of all TB cases, $1 \%-3 \%$ show skeletal system involvement and $30 \%$ are HIV coinfected. Although the reported distribution of skeletal TB is majorly in the spine, followed by the hip, knee, and foot/ankle, the epidemiology of extrapulmonary TB and especially musculoskeletal TB remains largely unknown, particularly in areas with a high prevalence of the disease.

Methods: This is a retrospective study of a consecutive series of patients admitted to a tertiary care facility in an area with the highest prevalence of TB worldwide. TB was confirmed on tissue biopsy with polymerase chain reaction testing (Xpert for Mycobacterium tuberculosis and rifampicin resistance), culturing, or histological analysis. Data were analyzed regarding demographic information, location of the disease, HIV coinfections, and drug resistance.

Results: In all, 125 patients (44 children; 35\%) with a mean age of 27 years (range, 1-78 years) were included. Age peaks were observed at 5, 25, and 65 years. Spinal disease was evident in 98 patients (78\%). There were 66 HIV-negative (53\%) and 29 (23\%) HIVpositive patients, and in $30(24 \%)$, the HIV status was unknown. Five patients (4\%) showed MDR TB.

Conclusions: The age distribution was trimodal, spinal disease was predominant, MDR TB rate in our cohort was high, and a large portion of TB patients in our hospital were HIV coinfected. Hence, spinal services with sufficient access to operating facilities are required for tertiary care facilities in areas with a high TB prevalence.

Keywords: Spine; Bone and joint; Tuberculosis; HIV; Epidemiology

\section{Introduction}

Worldwide, 9 million new tuberculosis (TB) cases are annually reported; of these, approximately 1 million (13\%) occur in human immunodeficiency virus (HIV)- positive hosts [1]. South Africa is one of the countries with the highest prevalence of TB, with 860 cases per 100,000 people reported in 2013; most of the cases in this country show HIV coinfection [1]. In the Western Cape and Cape Town, the incidence of TB is even higher, with

\footnotetext{
Received Jul 19, 2016; Revised Oct 11, 2016; Accepted Nov 4, 2016

Corresponding author: Michael F. G. Held

Orthopaedic Research Unit, Department of Orthopaedic Surgery, Groote Schuur Hospital, University of Cape Town,

H49 Old Main Building, Groote Schuur Hospital, Observatory 7925, Cape Town, South Africa

Tel: +27-214066157, Fax: +27-21472709, E-mail: email.held@gmail.com
} 
1400 cases per 100,000 people [2].

The rate of extrapulmonary TB (EPTB) worldwide has reached $20 \%-40 \%$ (20\% in children), as reported in recent studies [3-5]. Young patients, females, and people of African or Asian origin seem to have a higher risk of developing EPTB [6,7]. Of cases with EPTB, 10\%-25\% have musculoskeletal TB $[1,8]$, leading to an estimated global prevalence of 19-38 million cases [9]. The most commonly affected site of infection is the spine (50\%-69\%), followed by the hip, knee, and ankle/foot (10\%-13\% each) [7]. Data on childhood musculoskeletal TB is very rare. At Red Cross Children's Hospital in Cape Town, the largest children's hospital in sub-Saharan Africa, approximately 20 children under the age of 12 years are treated for musculoskeletal TB each year (60\% in the spine, $20 \%$ in knee, $16 \%$ in hip, and less than $5 \%$ in ankle or upper limbs) [10].

One of the major challenges with TB control is multidrug resistance. The World Health Organization (WHO) estimates $3.5 \%$ of new cases and $20.5 \%$ of previously treated cases to show multidrug-resistant (MDR) TB. Although South Africa has one of the world's highest TB and MDR TB rates, the epidemiology for EPTB and especially musculoskeletal TB remains largely unknown. South Africa is a very diverse society with TB largely affecting the poor owing to poor socioeconomic conditions. These include high-density informal dwellings where people are vulnerable in inclement weather. Factors such as malnutrition and close contact with others showing active disease due to delayed diagnosis, frequent non-compliance with medical care, and co-existing HIV drive the promulgation of the disease. This failure to complete full medical therapy increases the multidrug resistance rate. In our population, we estimate $5 \%$ of musculoskeletal TB cases to show MDR TB [11], making a correct diagnosis on tissue biopsy imperative to identify resistance and initiate optimal treatment.

The aim of this study was to assess age distribution, infection site, incidence of TB/HIV coinfection, and rate of MDR TB in patients with musculoskeletal TB at our tertiary care hospitals in Cape Town.

\section{Materials and Methods}

This is a retrospective analysis of a consecutive series of patients with tissue-confirmed TB admitted to Red Cross Hospital and Groote Schuur Hospital in Cape Town, South Africa over a period of 18 months between March
2013 and December 2014. These hospitals service an area that has the highest prevalence and incidence of TB worldwide. The vast majority of our patients are from the lower socioeconomic class and a high percentage live in townships or informal settlements with high-density housing.

Owing to a high rate of MDR TB, local guidelines recommend that all cases of suspected musculoskeletal $\mathrm{TB}$ undergo tissue confirmation and resistance testing. Musculoskeletal TB is suspected when patients present with suspicious symptoms and signs (painful or swollen joints or back pain) in conjunction with constitutional symptoms (night sweats, loss of appetite, and weight loss). Chronic cough, history of TB contact, or HIV infection will increase our index of suspicion. Biopsies were performed under general anesthesia from radiologically predetermined areas of disease (Fig. 1). All tissue-confirmed TB cases were selected from a surgical database of orthopedic biopsies performed at our hospitals during the study period. Only a small subgroup of patients had a screen of associated extraosseous TB by means of gastric lavage or sputum testing. Thus, this information was not collected.

The study was approved by the human research ethics committee of the Faculty of Health Sciences, University of Cape Town. The study adhered to the Ethical Principles for Medical Research Involving Human Subjects outlined in the Helsinki Declaration of 1975 (revised 2013).

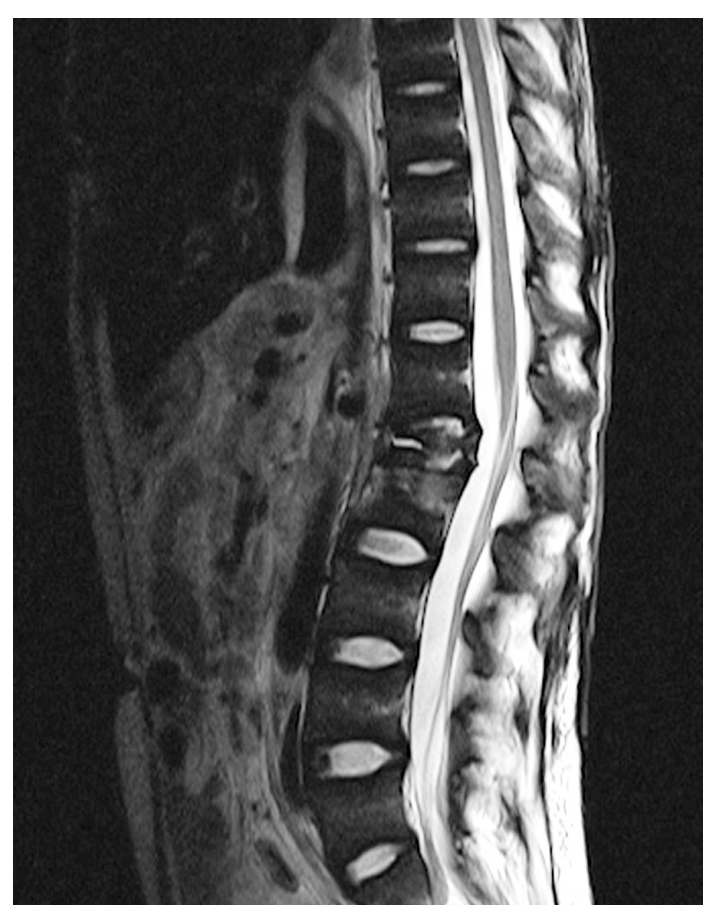

Fig. 1. Magnetic resonance imaging with spinal tuberculosis. 


\section{Definitions}

TB was defined as a positive TB culture, Xpert test for Mycobacterium tuberculosis and resistance to rifampicin (MTB/RIF) (Xpert MTB/RIF assay, Cepheid, Sunnydale, CA, USA), or histology-positive for acid-fast bacilli with granulomatous infection or apparent Langerhans cells. TB negativity was assumed if an alternative diagnosis was confirmed on tissue diagnosis or if culture, Xpert, or histology were negative for TB and patients improved without TB treatment. Improvement was assessed for at least 1 month after treatment was commenced by means of decreasing erythrocyte sedimentation rates and improvement of clinical symptoms. Patients aged 18 years or older were considered adults. Children were defined as younger than 18 years of age.

\section{Diagnostic tests}

The samples were subjected to standard diagnostic tools for testing of TB and polymerase chain reaction analysis (Xpert MTB/RIF assay, Cepheid). Xpert MTB/RIF is an automated diagnostic test that simultaneously detects $\mathrm{TB}$ and rifampicin resistance. It has recently been shown to have a high sensitivity and specificity pertaining to spine TB [11].

WHO defines standard tissue microscopy and liquid culture as standard testing tools for musculoskeletal TB [1]. BACTEC MGIT 960 by BD Diagnostic Systems (Sparks, MD, USA) was used for culture. Direct microscopy for TB was performed with a Ziehl-Nielson (ZN) stain as well as a hematoxylin and eosin stain. The $\mathrm{ZN}$ stain was used to identify acid-fast bacilli and HE stain for necrotizing granulomata. TB was defined as a positive Xpert MTB/RIF test, culture, or histology. Multidrug resistance was defined as resistance to isoniazid and rifampicin, with or without resistance to other first-line drugs, as per definition by the WHO. Genotype MTBDRplus or Genotype Mycobacterium line probe assays (Hain Lifescience, Nehren, Germany) were used to confirm resistance to isoniazid and rifampicin in culture-positive samples. A trained technician blinded to the culture and histopathology results processed the Xpert MTB/RIF assay. The histopathology slides were reviewed by a specialist pathologist who was blinded to the culture results but not to the Xpert MTB/RIF results or clinical data.

Based on the recommendations of the South African
Department of Health HIV counseling and Testing Policy Guidelines, HIV testing is offered to all patients at our hospital. For this study, the HIV status was determined by means of testing for HIV antibodies, antigens, or enzymes in a blood sample. The laboratory reports were accessed through the National Health Laboratory System.

The treatment and outcome were not assessed in this study. Patients were treated with 9 months of a daily weight-related four-drug combination medication containing isoniazid, rifampicin, pyrazinamide, and ethambutol. The dose was maintained throughout and not reduced after the first 2 months, as in the pulmonary TB protocol. MDR TB was treated according to the sensitivity screen with drugs such as amikacin, kanamycin, ofloxacin, ethionamide, and ethambutol. In spinal TB, medical therapy was often combined with surgical debridement and stabilization.

\section{Statistical evaluation}

Data was analyzed according to the location of the disease, patients' age, and HIV status. For differences between groups, the two-tailed Fisher exact test was used. All statistical analyses were conducted using SAS ver. 9.4 (SAS Institute, Inc., Cary, NC, USA).

\section{Results}

Of 303 consecutive biopsy patients, 125 patients (56 males, 44.8\%) had tissue-confirmed TB and were included in the study. Of this, group 81 patients were adults with a mean age of 27 years (range, 18-78 years). In the 44 children (35\%), the mean age was 5.6 years (range, 1-17 years). Three age peaks were identified at 5, 25, and 65 years (Fig. 2).

Of the 125 patients, 98 (78\%) had TB of the spine. The remaining 27 (21.6\%) had extraspinal disease (Table 1). Of the 44 patients under 18 years of age, 26 (59\%) suffered from spine TB, whereas 18 (41\%) showed extraspinal disease. Only 1 patient had TB of the bone (0.8\%). Of the 81 patients older than 18 years, 72 had spine TB, which was significantly higher than the number of patients with extraspinal disease $(p<0.001)$.

There was no difference in infection sites between HIVpositive and -negative patients $(p=0.31)$. Among the HIV coinfected patients, 25 of 29 (86\%) presented with TB of the spine, whereas only four (14\%) showed extraspinal 


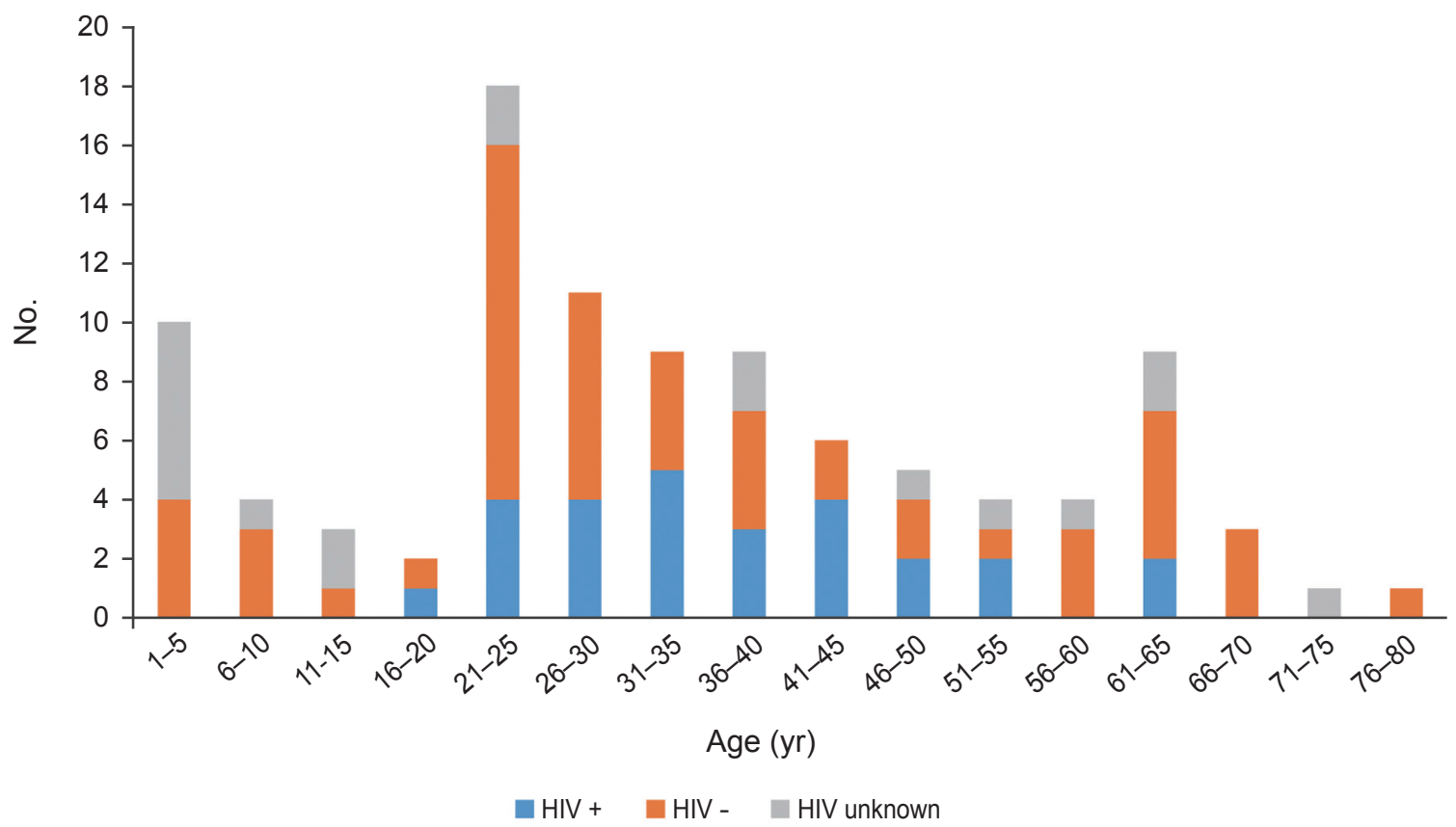

Fig. 2. Age distribution of tuberculosis and human immunodeficiency virus (HIV) co-infections.

Table 1. Distribution of infection sites and age group (children vs. adults) in 125 patients

\begin{tabular}{lccc} 
Infection site & Total & Children & Adults \\
Spine & $98(78)$ & $26(59)$ & $72(89)$ \\
\hline Hip & $6(5)$ & $4(9)$ & $2(2)$ \\
\hline Knee & $9(7)$ & $8(18)$ & $1(1)$ \\
\hline Foot/Ankle & $1(1)$ & 0 & $1(1)$ \\
\hline Shoulder & $3(2)$ & $2(5)$ & $1(1)$ \\
\hline Elbow & $5(4)$ & $2(5)$ & $3(4)$ \\
Wrist & $1(1)$ & 0 & $1(1)$ \\
\hline Others & $2(2)$ & $2(5)$ & 0 \\
\hline Total & $125(100)$ & $44(35.2)$ & $81(64.8)$ \\
\hline
\end{tabular}

Values are presented as number (\%).

disease (Table 2). Sixty-six patients (53\%) were HIVnegative and 29 (23\%) HIV-positive. In 30 (24\%), the HIV status was unknown. Five of the patients (4\%) were found to be MDR (Table 3).

\section{Discussion}

This is the first large study of tissue-confirmed musculoskeletal TB to describe patients' age distribution, location of disease, HIV coinfection, and multidrug resistance.

The reported peak age of adult patients with musculoskeletal TB ranges from 45 to 60 years [7]. Although some studies report a bimodal age distribution [12], we found a distribution with three peaks, i.e., in the first, third, and sixth decade of life. It is well-accepted that young children are commonly affected by TB in areas with a high disease prevalence [13], whereas the second peak, between 20 and 30 years of age, is often reported to be related to HIV or the influx of immigrants in developed countries [7]. The third peak, between 60 and 70 years of age, is probably attributed to increased life expectancy, malnutrition, and comorbid chronic medical conditions (possibly diabetes and pulmonary), resulting in an age-associated immune vulnerability [14]. South Africa is unique in having both 
Table 2. Distributions of infection sides between HIV positive, negative and patients with unknown HIV status ( $n=125)$

\begin{tabular}{lccc} 
Infection site & HIV positive & HIV negative & HIV unknown \\
Spine & $25(20)$ & $53(42)$ & $20(16)$ \\
Hip & $1(0.8)$ & 0 & $5(4)$ \\
\hline Knee & 0 & $5(4)$ & $4(3.2)$ \\
\hline Foot/Ankle & 0 & $1(0.8)$ & 0 \\
\hline Shoulder & $2(1.6)$ & $1(0.8)$ & 0 \\
Elbow & 0 & $4(3.2)$ & $1(0.8)$ \\
Wrist & 0 & $1(0.8)$ & 0 \\
Others & $1(0.8)$ & $1(0.8)$ & 0 \\
\hline Total & $29(23)$ & $66(53)$ & $30(24)$ \\
\hline
\end{tabular}

Values are presented as number (\%).

HIV, human immunodeficiency virus.

Table 3. Characteristics of patients diagnosed with multidrug resistant tuberculosis

\begin{tabular}{lclc} 
Area of biopsy & Age (yr) & Sex & HIV status \\
Bone & 21 & Male & Negative \\
Spine & 53 & Female & Positive \\
Spine & 36 & Male & Positive \\
Spine & 29 & Female & Positive \\
Spine & 22 & Male & Negative \\
\hline
\end{tabular}

HIV, human immunodeficiency virus.

a developed-country population with a higher life expectancy and a developing-country population with highdensity housing and a high prevalence of HIV.

The distribution of infection sites in patients younger than 18 years of age (Table 1) is comparable with previously reported data [15-20]. In contrast, the proportion of spine TB was much larger in our cohort than that previously described in patients over 18 years of age (Table 1) $[7,19]$. This might be owing to the fact that some patients with extraspinal disease did not warrant referral by their regional hospitals and were therefore not included in this study. In the referring hospitals, suspected joint or bone infections are managed by general orthopedic specialists, with sufficient access to theatre, but no spinal surgical services are provided at these facilities. This emphasizes the requirement for an adequate spinal service to treat TB patients at large referral centers in areas with a high disease prevalence.

We found a higher rate of HIV/TB coinfection (23\%) in our cohort than that reported in the rest of the world (13\%). Most patients with TB/HIV coinfections were be- tween 20 and 30 years of age (Fig. 1). Our MDR TB rate of $4 \%$ is also higher than that reported in American and Indian studies, where only $0.9 \%$ MDR cases of all patients present with EPTB [5]. In our cohort, three of the five MDR cases were HIV-positive and four cases had spine TB (Table 3). A regional and national TB Control Program exists with the overall objective of reducing TB mortality and morbidity and preventing drug resistance [20]. Unfortunately, deficits in universal care and HIV epidemics hamper disease control and allow increased MDR TB in the country [21].

Despite the limitation of our study that $20 \%$ of our patients did not have a known HIV status, it is in line with the WHO data for the African region estimating that $24 \%$ of patients have an unknown HIV status [1]. For future, we hope that improved information and counseling services will decrease the number of patients unaware of HIV testing or declining HIV testing. Conditions leading to immunocompromization other than HIV, such as diabetes, have not been included because this information was not consistently recorded in the notes. Also, for future 
studies we should include all referring hospitals in an area of high TB prevalence to avoid preselecting spinal TB due to local referral patterns.

\section{Conclusions}

In conclusion, the age distribution identified three peaks, i.e., at 5, 25, and 65 years of age. Seventy-eight percent of cases presented with spine TB. The HIV coinfection rate was $23 \%$. Four percent of cases had MDR TB. This confirms that referral hospitals in areas with a high $\mathrm{TB}$ prevalence require spinal resources to perform biopsies for detecting MDR TB. Another focus must be to improve access to tertiary care hospitals and specialist care for children with musculoskeletal TB in their first decade of life, wherein the first peak was observed. Moreover, especially for TB patients in their third decade of life, HIV testing is essential to decrease morbidity and mortality with adequate antiretroviral therapy.

\section{Conflict of Interest}

No potential conflict of interest relevant to this article was reported.

\section{References}

1. World Health Organization. Global tuberculosis report 2014 [Internet]. Geneva: World Health Organization; c2014 [cited 2016 Aug 29]. Available from: http://apps.who.int/iris/bitstream/10665/ 137094/1/9789241564809_eng.pdf?ua=1.

2. Lawn SD, Bekker LG, Middelkoop K, Myer L, Wood R. Impact of HIV infection on the epidemiology of tuberculosis in a peri-urban community in South Africa: the need for age-specific interventions. Clin Infect Dis 2006;42:1040-7.

3. Gunal S, Yang Z, Agarwal M, Koroglu M, Arici ZK, Durmaz R. Demographic and microbial characteristics of extrapulmonary tuberculosis cases diagnosed in Malatya, Turkey, 2001-2007. BMC Public Health 2011;11:154.

4. Jutte PC, van Loenhout-Rooyackers JH, Borgdorff MW, van Horn JR. Increase of bone and joint tuberculosis in The Netherlands. J Bone Joint Surg Br 2004;86:901-4.

5. Peto HM, Pratt RH, Harrington TA, LoBue PA, Arm- strong LR. Epidemiology of extrapulmonary tuberculosis in the United States, 1993-2006. Clin Infect Dis 2009;49:1350-7.

6. Wang X, Yang Z, Fu Y, Zhang G, Zhang Y. Insight to the epidemiology and risk factors of extrapulmonary tuberculosis in Tianjin, China during 2006-2011. PLoS One 2014;9:e112213.

7. Johansen IS, Nielsen SL, Hove M, et al. Characteristics and clinical outcome of bone and joint tuberculosis from 1994 to 2011: a retrospective register-based study in Denmark. Clin Infect Dis 2015;61:554-62.

8. Anley CM, Brandt AD, Dunn R. Magnetic resonance imaging findings in spinal tuberculosis: Comparison of HIV positive and negative patients. Indian J Orthop 2012;46:186-90.

9. Malaviya AN, Kotwal PP. Arthritis associated with tuberculosis. Best Pract Res Clin Rheumatol 2003; 17:319-43.

10. Maqungo S, Oleksak M, Dix-Peek S, Hoffman EB. Tuberculosis of the foot and ankle in children. SA Orthopaedic J 2012;11:23-8.

11. Held M, Laubscher M, Zar HJ, Dunn RN. GeneXpert polymerase chain reaction for spinal tuberculosis: an accurate and rapid diagnostic test. Bone Joint J 2014; 96:1366-9.

12. Colmenero JD, Jimenez-Mejias ME, Reguera JM, et al. Tuberculous vertebral osteomyelitis in the new millennium: still a diagnostic and therapeutic challenge. Eur J Clin Microbiol Infect Dis 2004;23:47783.

13. Watts HG, Lifeso RM. Tuberculosis of bones and joints. J Bone Joint Surg Am 1996;78:288-98.

14. Mori T, Leung CC. Tuberculosis in the global aging population. Infect Dis Clin North Am 2010;24:75168.

15. Dix-Peek SI, Vrettos BC, Hoffman EB. Tuberculosis of the elbow in children. J Shoulder Elbow Surg 2003; 12:282-6.

16. Hoffman EB, Allin J, Campbell JA, Leisegang FM. Tuberculosis of the knee. Clin Orthop Relat Res 2002;(398):100-6.

17. Campbell JA, Hoffman EB. Tuberculosis of the hip in children. J Bone Joint Surg Br 1995;77:319-26.

18. Lee AS, Campbell JA, Hoffman EB. Tuberculosis of the knee in children. J Bone Joint Surg Br 1995; 77:313-8.

19. Sandher DS, Al-Jibury M, Paton RW, Ormerod LP. 
Bone and joint tuberculosis: cases in Blackburn between 1988 and 2005. J Bone Joint Surg Br 2007;89: 1379-81.

20. Department of Health, Western Cape Government. TB Control Programme, Western Cape [Internet].
Cape Town: Western Cape Government; c2014 [cited 2016 Aug 29]. Available from: https://www.westerncape.gov.za/service/tb-control-programme.

21. Weyer K. Case study: South Africa. Bull World Health Organ 2007;85:391-2. 